\title{
La réception de la sexualité dans les magazines pour adolescentes. Entre adhésion et contestation
}

Reception of Sexual Content in Teenage Girls' Magazines : Between Adherence and Contestation

Marie-Ève Lang

\section{OpenEdition}

Journals

Édition électronique

URL : https://journals.openedition.org/questionsdecommunication/6692

DOI : 10.4000/questionsdecommunication.6692

ISSN : 2259-8901

Éditeur

Presses universitaires de Lorraine

Édition imprimée

Date de publication : 1 septembre 2012

Pagination : 247-268

ISBN : 978-2-8143-0120-7

ISSN : $1633-5961$

\section{Référence électronique}

Marie-Ėve Lang, "La réception de la sexualité dans les magazines pour adolescentes. Entre adhésion et contestation », Questions de communication [En ligne], 21 | 2012, mis en ligne le 01 septembre 2014 consulté le 27 octobre 2022. URL : http://journals.openedition.org/questionsdecommunication/6692 ; DOI : https://doi.org/10.4000/questionsdecommunication.6692 


\title{
$>$ NOTES DE RECHERCHE
}

\section{MARIE-ÈVE LANG}

Groupe de recherche sur les images et les représentations sociales

Université Laval, Canada

marie-eve.lang1@ulaval.ca

\section{LA RÉCEPTION DE LA SEXUALITÉ DANS LES MAGAZINES POUR ADOLESCENTES. ENTRE ADHÉSION ET CONTESTATION}

\begin{abstract}
Résumé. - Dans un contexte d'hypersexualisation des produits médiatiques au Québec, nous avons voulu savoir de quelle façon le contenu à caractère sexuel du magazine pour adolescentes controversé Adorable était reçu par les lectrices. Nous avons rencontré 14 jeunes franco-canadiennes âgées entre 15 et 17 ans afin de déterminer comment ce type de textes les intéressait et ce qu'elles recherchaient dans ces articles, c'est-à-dire dans quelle mesure ceux-ci jouaient un rôle dans leur apprentissage de la sexualité. Alliant une méthode inspirée du système d'annotation élaboré par Sylvie Debras (2003) à des entretiens individuels et de petits groupes, notre étude a montré que : I) les articles à caractère sexuel ont été appréciés à $80 \%$;2) la fonction dominante remplie par ceux-ci a en effet été l'apprentissage, suivie de près par celle de plaisir ; et 3) la réception principale a surtout été de type dominante, mais les réceptions négociée et oppositionnelle ont également été bien présentes.
\end{abstract}

Mots clés. - Études de réception, hypersexualisation, magazines, adolescentes, Québec. 
$\mathrm{D}$ epuis la fin des années 90, on s'interroge au Québec sur les implications et les effets d'un nouveau phénomène de société : l'hypersexualisation des jeunes filles. Terme à « connotation fortement négative » (Caron, 2009 : 29), l'hypersexualisation désigne à la fois une mode vestimentaire particulièrement sexy, des pratiques sexuelles jugées précoces, « une "pornoïsation" de la culture » (ibid. : 28-29) en général et une image spécifique de la femme dans les médias. Toutefois, l'alarmisme ambiant initial se serait transformé dans les années 2000 en véritable « panique morale » dans les médias et dans le discours public, selon l'interprétation de la chercheuse Caroline Caron. Pour mieux comprendre le phénomène de l'hypersexualisation et ses implications, de nombreux journalistes et chercheurs se sont mis à étudier certains contenus médiatiques nordaméricains, dont les magazines pour adolescentes. II s'en est suivi beaucoup d'études de contenu, mais très peu d'études de réception, pourtant essentielles pour comprendre le point de vue des principales intéressées. Dans le but de donner la parole aux jeunes femmes lectrices de ces magazines, nous avons mené de 2007 à 2009 une analyse de réception des textes à caractère sexuel contenus dans le magazine québécois pour adolescentes Adorable. Parmi les magazines pour adolescentes ayant été produits au Québec durant les dernières années, Adorable est de loin celui ayant obtenu la plus mauvaise presse, en grande partie parce que son contenu est ouvertement très sexuel - environ le tiers de ses pages est consacré à ce thème. La sexualité constitue sa principale accroche de vente et joue un rôle central dans son contenu, qui présente une forte tendance à « insister sur les relations sexuelles » (Caron, 2003b : 91 ; 131 ; Bouchard, Bouchard, 2003 : I 4- I 5). En outre, Adorable a plus d'une fois suscité la controverse. En 2002, son « Guide 100 \% sexe : 99 trucs coquins pour amener votre mec au $7^{\mathrm{e}}$ ciel » a provoqué une petite commotion au sein du débat public qui émergeait alors au sujet de l'hypersexualisation des jeunes filles et a fait le tour des médias (Caron, ibid. : 9). En réponse aux critiques qui lui ont été adressées, l'équipe du magazine Adorable s'est expliquée en affirmant avoir changé de public-cible et qu'elle s'adressait depuis peu aux jeunes femmes'. Cependant, si le public-cible a changé en 2002, le véritable lectorat, lui, est vraisemblablement resté le même, du moins en partie. D'ailleurs, nous avons pu trouver, pour les fins de notre recherche, une quinzaine de participantes adolescentes, âgées entre 15 et 17 ans, ayant déjà lu Adorable ou le lisant toujours.

\footnotetext{
Un avis en « post-post-scriptum » du billet de la rédactrice dans le numéro de juin 2007 a rappelé ce changement de cible, alors que le magazine a été fusionné pendant quelque temps avec la publication Corps et âme, une revue pour femmes « trait[ant] de la sexualité, [de] la vie de couple et de l'amour » (Trudeau, 2007: 4) et qu'il prenait, quelques mois plus tôt, une partie du titre de son pendant masculin Summum (un magazine pour hommes axé sur la sexualité) pour devenir Adorable au Summum. Maintenant nommé Summum Girl, le magazine a conservé, au fil des ans, le même contenu sexualisé, contenu qu'on a même promis « beaucoup plus "chaud" » (ibid.) en 2007.
} 


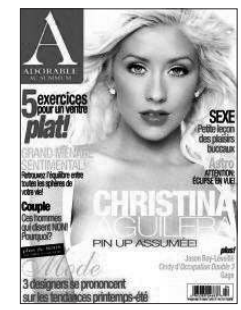

Illustration I. Page de couverture d'un numéro d'Adorable.

Même si l'on connaissait la grande proportion d'articles traitant de sexualité dans ce magazine, ce que les jeunes lectrices faisaient de ce contenu était peu clair. Le lisaient-elles dans le but de s'informer au sujet de la sexualité ? Le lisaientelles plutôt pour se divertir, s'amuser ? Y prêtaient-elles même une attention particulière ? En effet, il était possible et même probable que les lectrices lisent Adorable pour s'informer au sujet de la sexualité puisque, comme l'ont indiqué Robert T. Francoeur et Raymond J. Noonan (2004 : 135-139), les adolescentes cherchent parfois à s'informer et s'éduquer au sujet de la sexualité par la lecture d'articles de magazines au détriment d'autres sources comme les parents et les éducateurs spécialisés. Cependant, il était aussi possible que, à l'opposé, les lectrices feuillettent ce magazine sans trop faire de cas des articles au contenu sexualisé. Selon Joke Hermes (1995: 29), les magazines sont d'ailleurs des publications consultées de façon plus ou moins concentrée, et qu'on peut aisément mettre de côté (« easily put down »). Pour combler le manque de connaissances sur les habitudes de lecture des lectrices d'Adorable et pour déterminer dans quelle optique sont lus les textes traitant de sexualité que contient le magazine, nous avons mené une étude de réception structurée en trois plans distincts, chacun visant à répondre à l'une de ces trois questions :

I. À quel degré les participantes s'intéressent-elles au contenu sexuel du magazine?

2. Quelle(s) fonction(s) la lecture des textes traitant de sexualité remplit-elle chez elles?

3. Quelle est leur opinion de ces textes?

Le but de cet article est de présenter le cadre théorique, la méthode et les résultats de notre analyse.

\section{Cadre théorique}

Les études de réception s'inscrivent dans le courant des Cultural Studies dont le coup d'envoi a été donné en grande partie par Stuart Hall (1980; 2007). Son 
modèle théorique du codage/décodage qui suppose un décalage entre les significations que les publics tirent des produits médiatiques et celles que les producteurs visent à « encoder », a jeté les bases d'une reconnaissance du rôle actif des récepteurs dans la création des significations des produits de la culture populaire. Pour illustrer cette absence de correspondance parfaite, Stuart Hall a défini trois réceptions-types, à savoir : les réceptions « oppositionnelle », « négociée » et « dominante » (ou « préférée »). Selon lui, la réception dominante correspond à une lecture allant dans le même sens que celle « encodée » par les producteurs, c'est-à-dire que les récepteurs comprennent le sens initial d'une production et y adhèrent. Cependant, lors d'une lecture oppositionnelle, le lecteur peut comprendre parfaitement le sens littéral d'un texte et ses connotations, mais il se positionne tout à fait à l'opposé de ces dénotations et confère au message une signification alternative. Enfin, la position négociée, se situe à cheval entre les deux autres types de réception : le lecteur adhère aux grandes lignes, abstraites, d'un produit médiatique, mais se réserve le droit ou l'occasion de l'adapter à sa propre situation, se permettant des contradictions et des réadaptations. Selon Liesbet Van Zoonen (1994:8), le fondement théorique de bien des études de réception faites sur les femmes s'appuieraient sur « une légère variation » de la théorie du codage/décodage élaborée par Stuart Hall, c'est-à-dire sur l'idée qu'il y aurait, de façon fort probable, un écart entre les codes de la source et ceux des récepteurs en ce qui concerne les messages médiatiques (voir aussi Stevenson, 2002 : 108). Janice Radway (1984) est un exemple parfait d'une étude féministe s'appuyant sur cette variation de la théorie du codage/décodage. Combinant une analyse de contenu et une analyse de la réception des romans populaires dits «à l'eau de rose » (de type Harlequin), elle s'est intéressée à la construction du sens de ces romans chez les lectrices assidues. Par le moyen de questionnaires et d'interviews, elle a interrogé des femmes américaines de la classe moyenne faisant partie d'un club de lecture sur leurs goûts, leurs usages de ces livres et leurs conceptions des récits. Elle s'est rendu compte qu'il y avait une différence notable entre la construction du récit chez les lectrices et l'interprétation que faisaient les chercheuses de ces mêmes textes. Ces romans populaires étaient lus dans une perspective « d'évasion de leur fardeau quotidien » (Van Zoonen, 1994: I I0- I I I), leur lecture agissant en quelque sorte comme une activité « compensatoire », « procurant aux femmes l'attention et les soins dont elles manquaient dans leur vie de tous les jours », alors même que les chercheuses, ne s'intéressant qu'au contenu, n'y voyaient qu'une série de clichés issus du patriarcat. L'étude de Janice Radway démontre deux choses importantes : d'abord, que les lectrices ou les réceptrices ne lisent pas ou ne reçoivent pas les contenus médiatiques nécessairement « comme le font les chercheuses » (Caron, 2003b : 124- 125) et, qu'ainsi, pour véritablement rendre compte du sens des contenus populaires chez les femmes (ou chez les récepteurs en général), il est nécessaire de s'intéresser aux interprétations du public même (Van Zoonen, ibid. : 106) ; et ensuite, que l'action de lecture en elle-même constitue une source de plaisir : le simple fait de prendre et de choisir un livre contribuait, pour les participantes de Janice Radway, à ce plaisir. Le fait de 
lire la romance était pour elles tout aussi important, sinon plus, que les significations de cette romance (Radway, ibid. : 86 et sq.). Dans le champ des études portant sur les magazines, et en particulier sur les magazines pour adolescentes, les recherches sur la réception se sont faites plutôt rares. D'abord, parce que les magazines pour adolescentes, surtout au Québec, sont en soi un phénomène relativement nouveau : le premier magazine pour jeunes filles au Québec a vu le jour en 1980 ; les autres sont apparus en vaste majorité vers la fin des années 90 (Caron, ibid. : 20). Ensuite, comme cela a été le cas pour les recherches sur la consommation de contenus médiatiques populaires par les femmes, les études sur les magazines pour adolescentes se sont d'abord souciées du contenu avant de s'intéresser à ce que les jeunes filles faisaient de ce contenu. Enfin, analysées souvent sous l'angle des théories de la socialisation, les études de contenu ont, comme avec les études sur les femmes, plutôt tenté de dégager une idéologie « dominante » qui serait cachée aux jeunes filles et dont elles seraient « victimes »². En effet, plus souvent qu'autrement, puisque les analyses de contenu dépeignaient les magazines comme un univers « normatif et prescriptif qui assigne des rôles sexuels contraignants » (Cyr, 1998: 16), il s'en fallait de peu pour qu'un raccourci méthodologique soit fait et que les lectrices soient représentées comme étant « dupées » par les textes, surtout que celles-ci, contrairement aux lectrices de magazines pour femmes, sont mineures et, par conséquent, bien souvent considérées comme ayant moins d'esprit critique. Elizabeth Frazer (1987) a été l'une des premières à démontrer que les jeunes lectrices étaient rarement victimes des textes et à postuler que les produits médiatiques offrent une polysémie de significations plutôt qu'un « "vrai sens" (unique et monolithique) que seuls les universitaires peuvent déceler à l'aide d'une analyse méticuleuse des messages »(Caron, 2003a : 25). En effet, son étude, s'appuyant sur des entrevues avec des lectrices âgées de 13 à 17 ans, a démontré que les jeunes lectrices «n'interprètent pas nécessairement les messages véhiculés par le texte de la même façon dont celui-ci [a été] construit » (ibid. : 25-26). Au Canada, très peu d'études de réception ont été menées sur les magazines pour adolescentes. Au Canada anglais, Dawn Currie (1999) a démontré que les lectrices, bien qu'elles semblaient généralement adhérer à l'idéologie dominante des textes, pouvaient faire preuve de cynisme ou d'esprit critique envers certains contenus précis, notamment dans le cas des lettres envoyées à l'équipe éditoriale demandant un conseil ou dans le cas certaines publicités jugées irréalistes. Mais au Québec comme dans le reste du Canada français, du moins au moment d'effectuer notre recherche, très peu d'études de réception avaient été effectuées

\footnotetext{
2 Par exemple, la première étude d'A. McRobbie (1991 : 81-82), bien que présentant un volet " réception », a plutôt présenté « une critique systématique de Jackie comme un système de messages et de significations porteur d'une certaine idéologie », et non les résultats d'une réelle analyse de la réception. Cette idée de l'auteure d'une force idéologique très puissante, de même son « sentiment ouvertement hostile au magazine », le fait de " privilégier sa propre lecture du magazine plutôt que de s'appuyer sur une analyse plus formelle » (Stevenson, 2002 : 108) et plus encore l'absence très remarquée de l'analyse de la réception faite par les lectrices lui ont valu de nombreuses critiques.
} 
sur les magazines québécois pour adolescentes, et encore moins sur les textes à caractère sexuel qu'ils contiennent; et ce, même si les médias et la société en général s'entendaient pour dire que la « teneur » en sexualité de ces magazines était inquiétante. Notre étude sur la réception des textes à caractère sexuel par les lectrices ne pouvait donc que s'avérer hautement pertinente, vu le faible nombre d'études canadiennes conduites en ce sens, particulièrement au Canada français. Par ailleurs, puisque la voix des adolescentes dans l'espace public est trop souvent, selon Caroline Caron (2007), « soit marginalisée, soit stigmatisée, soit ignorée », nous avons voulu, comme elle, « donner une voix aux adolescentes, de manière à insérer cette voix dans les débats [publics], de faire entendre une réalité qui est autrement ignorée ». L'une de nos motivations à effectuer une étude de réception auprès des adolescentes était donc de contribuer à donner une légitimité de parole aux adolescentes, qui ont été exclues, d'une part, des débats publics qui les concernent au premier chef (notamment celui sur l'hypersexualisation) et, d'autre part, de l'ensemble de la recherche actuelle sur la sexualité et les magazines, qui ont en général négligé de s'interroger sur le sens que construisaient les réceptrices de ce type de contenus ${ }^{3}$.

\section{Méthode}

Comme nous l'avons mentionné en introduction, notre recherche est organisée en trois plans, qui sont eux-mêmes motivés par trois catégories de questions fondamentales.

\section{Le plan de l'intérêt}

Dans quelle mesure les textes qui traitent de sexualité intéresse-t-ils les lectrices? Plus précisément, ces textes les intéressent-elles plus, moins ou autant que les textes du même magazine mais traitant d'autres thèmes, tels le vedettariat, l'actualité, la mode et la beauté ? D'abord, quels sont les articles et les sections du magazine qui les intéressent le plus, et quelles sont les images qu'elles préfèrent? Ces textes et ces images sont-ils en lien avec la sexualité? Certains de ces textes sont-ils tout simplement ignorés? Lesquels?

\section{Le plan de la fonction des textes}

Quelles fonctions la lecture des articles à caractère sexuel du magazine Adorable remplit-elle chez les lectrices? En d'autres termes, que recherchent-elles dans ces articles? Les lisent-elles dans une optique de loisir ou plutôt d'accès à la connaissance? Ces fonctions remplies par ces textes sont-elles différentes de celles des textes ne discutant pas de sexualité ? Ce que nous nommons

\footnotetext{
${ }^{3}$ Pour une description du concept de voix en sciences sociales, voir L.-F. Rakow, L.-A. Wackwitz (2005) et O.Voirol (2005a ; 2005b).
} 
ici « fonctions » fait référence à la théorie des « usages et gratifications », courant dont Paul Lazarsfeld a donné le coup d'envoi dans les années 60 et qui cherche à déterminer ce que les individus « font » des médias, en leur permettant d'adapter les contenus à leurs besoins (Lazarsfeld, Berelson, Gaudet, 1968).

3. Le plan de la réception en tant que telle (opinion)

Quelle opinion ont-elles des textes traitant de sexualité ? Plus particulièrement, dans quelle mesure démontrent-elles la capacité de porter un regard critique sur ces textes ou les images qu'ils contiennent? Les réactions à l'ensemble des articles laissent-elles croire à une acceptation de l'idéologie du magazine, à une ignorance de cette idéologie, à une contestation de celle-ci ou bien à une réception négociée (ambivalente) ? Cette réception diffère-t-elle lorsque l'on considère séparément les articles à caractère sexuel ?

Au terme de l'analyse de ces trois plans, il résulte une vision globale mais rigoureuse et pointue de la relation qu'entretiennent les participantes avec les textes à caractère sexuel contenus dans le magazine Adorable, qui permet de répondre de façon riche et complète à la question de recherche originale, qui pourrait être formulée ainsi : comment les jeunes filles franco-canadiennes qui lisent des magazines pour adolescentes reçoivent-elles les textes à caractère sexuel qu'ils contiennent? Chacun de ces plans de recherche appellent une méthode différente de collecte de données. À l'instar de Sylvie Debras (2003), nous avons donc combiné une méthode particulière de collecte de données quantitatives visant à mesurer l'intérêt porté aux articles (appelée « crayon en main ») à une méthode plus classique d'entretiens qualitatifs. La méthode « crayon en main », utilisée par Sylvie Debras dans le cadre de sa recherche sur les habitudes de lecture des quotidiens par des hommes et des femmes, consistait à demander aux participants « de surligner et d'annoter les articles selon leur niveau de consultation : lecture des titres seuls, lecture rapide du texte ou lecture intégrale ; on devait aussi noter sur les journaux la date, l'heure, la durée de lecture et l'appréciation des articles » (Lebel, 2004 : 388). Cette méthode, « très productive » (ibid.), permet d'obtenir un portrait riche de la relation qu'entretient le lecteur avec le texte. Dans notre cas, la méthode est légèrement différente de celle développée par Sylvie Debras et adaptée à l'âge des répondantes de même qu'aux besoins de la recherche. Après avoir recruté quatorze participantes âgées entre 15 et 17 ans $^{4}$ dans des polyvalentes du Québec et du Nouveau-Brunswick (la seule condition de participation étant d'avoir déjà lu Adorable dans le passé ou de le lire présentement, afin de ne pas imposer une lecture auxquelles elles

\footnotetext{
${ }^{4}$ Les participantes étaient toutes de jeunes franco-canadiennes d'origine caucasienne appartenant à la classe moyenne. Elles vivaient également toutes en région. Certaines d'entre elles lisaient plus que d'autres et avaient de meilleures notes à l'école. Cependant, en raison du petit nombre de participantes, notre intention première n'était pas de généraliser des comportements de lecture en fonction de certaines données sociodémographiques, mais bien d'observer leurs réactions générales envers le magazine. Toutefois, pour obtenir de plus amples renseignements à cet égard, voir M.-E. Lang (2009).
} 
ne seraient pas déjà habituées), nous avons remis à chacune trois exemplaires du magazine Adorable. Ceux remis étaient les mêmes pour chacune des participantes et constituaient des numéros choisis au hasard dans l'année courante (il est possible d'obtenir des copies de numéros déjà parus auprès du distributeur). On leur a également remis des stylos colorés avec l'instruction d'encercler le titre des articles lus ou non lus selon un code couleur: « article non lu », « peu intéressant », « intéressant » ou « très intéressant ». On leur a également demandé d'encercler leur image préférée dans le lot des trois magazines. Cette méthode a permis de quantifier l'intérêt qu'a suscité chaque article - et donc chaque section - des trois magazines remis aux participantes. Elle a aussi permis de déterminer quelles images étaient préférées - question dont la réponse peut notamment être utilisée pour entamer la discussion des articles lors des entretiens. Nous avons ensuite alloué plusieurs semaines aux participantes pour lire les trois revues, après quoi elles ont été invitées à participer à une entrevue individuelle ou à un groupe de discussion de leur choix. D'une façon générale, la méthode privilégiée par les études de la réception des contenus de la presse écrite est celle des groupes de discussion, des focus groups ou de l'entrevue semi-dirigée, souvent doublée ou précédée d'une mini-analyse documentaire servant de point de départ aux entrevues ou aux groupes de discussion, ou encore d'appui à l'analyse $e^{5}$. Nous avons privilégié une technique de recherche semblable à celles utilisées par les recherches antérieures sur le sujet, c'est-à-dire une technique qui se situe à mi-chemin entre l'entrevue semi-dirigée et le groupe de discussion, soit l'entretien semi-dirigé de « petit groupe » composé entre 2 et 5 participants. Les raisons motivant ce choix sont les suivantes: d'abord, comme nous allions discuter de sexualité, nous craignions que des entrevues semi-dirigées faites de façon individuelle causent une certaine gêne chez les participantes et nuisent à la collecte de données. Ensuite, pour la même raison, nous avons tenu à ce que le groupe soit formé d'amies relativement proches, de façon à ce qu'elles soient déjà à l'aise entre elles. Des petits groupes de deux ou trois amies constituaient donc pour nous le format idéal. Par ailleurs, le fait d'utiliser une méthode à mi-chemin entre les deux, c'est-à-dire de créer un « petit » groupe de discussion dont le schéma est fondé sur celui recommandé pour l'entrevue individuelle, permet de poser beaucoup plus de questions, ce qui favorise l'interaction : en posant plus de questions, nous assurons un caractère conversationnel, dialogique, à l'entretien, le rendant plus naturel et faisant oublier, du moins en partie, le statut d'autorité du chercheur. L'enregistrement des entretiens montre d'ailleurs que les « mini-groupes » de discussion ont été très réussis en ce sens : la participante, qui trouve une plus grande reconnaissance de son statut de sujet, converse librement avec ses pairs, malgré leur petit nombre. L'entrevue de type face-à-face, au choix de l'adolescente, n'était toutefois pas exclue (en cas de malaise ou de simple préférence). Cette ouverture à plusieurs méthodes est en effet souvent pratiquée lorsque le sujet de l'étude est tabou ou délicat - c'est d'ailleurs la technique qu'a privilégiée Elizabeth A. Kissling (1998: 485) dans son étude sur l'expérience des premières menstruations où

\footnotetext{
${ }^{5}$ Voir par exemple l'étude de P. Bouchard et N. Bouchard (2005).
} 
les jeunes filles, en plus de pouvoir choisir entre l'entrevue individuelle ou en petits groupes, pouvaient décider de participer en présence ou non de leur mère. Comme l'entretien occupe une très grande place dans cette démarche, notre recherche est donc surtout qualitative. L'intérêt d'une démarche qualitative est dans ce cas très élevé car elle permet de laisser une large place à la subjectivité des répondantes et d'observer le « phénomène » à étudier dans son contexte naturel « plutôt que dans un contexte qu'on tenterait de neutraliser au départ pour en arriver à établir rapidement des généralisations » (Laperrière, 1997 : 367). II en résulte une approche plus compréhensive, complexifiée et, par conséquent, plus «approfondie » (ibid. : 367-37l). La « codification précise, consistante et exhaustive » des données résultant des entretiens, entre autres éléments, assure enfin « la concordance entre les observations empiriques et leur interprétation », ce qui pourvoit à la recherche une grande partie de sa rigueur et de sa fiabilité (ibid. : 386).

\section{Analyse des données de la méthode « crayon en main »}

L'analyse des données issues des marques de lectures laissées par les participantes s'est faite comme suit : à chaque couleur du code, nous avons fait correspondre une donnée chiffrée. Ainsi, au bleu, qui signifiait «article non lu », avons-nous attribué la note de zéro ; au vert, qui signifiait « article lu mais peu intéressant », on a conféré la note de un ; et ainsi de suite jusqu'à la note de trois (pour les articles jugés « très intéressants »). Tout article qui n'avait pas fait l'objet de marques de la part des participantes a été considéré comme non lu et a reçu la valeur de zéro. Une fois que chaque article ${ }^{6}$ ait reçu une valeur déterminée, les données ont été compilées et amenées à une moyenne pondérée sur trois, qui a elle-même été ramenée, pour faciliter l'analyse, en un taux sur I, qui représente le taux d'intérêt suscité par chaque article chez les participantes.

\section{Analyse des entretiens}

Si la méthode «crayon en main » a servi à l'analyse du taux d'intérêt (qui constitue le plan I de notre démarche), les entretiens qualitatifs ont pour leur part permis de répondre aux deux autres plans : les fonctions et la réception concernant les opinions. Tous les entretiens que nous avons menés ont été enregistrés en format audio et ont ensuite été rédigés textuellement, à la suite de quoi plusieurs lectures des transcriptions ont été effectuées de façon à repérer et à comptabiliser les différentes fonctions remplies par les textes. Le système catégoriel pour les fonctions a donc été construit a posteriori.

\footnotetext{
6 Nous n'avons pas considéré les publicités et les publireportages, qui, de toutes façons, ont été ignorés par les participantes (aucune marque de lecture ou presque). II peut s'agir d'un réel désintérêt pour les publicités ou encore du résultat de la façon dont ont été présentées les consignes de lecture, qui insistaient sur les articles.
} 
Nous avons procédé différemment pour l'analyse de la réception, dont les catégories ont été établies a priori. En effet, nous avons rendu opérationnel le concept de réception par une catégorisation en quatre différentes réactions : acceptation de l'idéologie du texte, contestation du texte, contestation partielle du texte et ignorance (c'est-à-dire que l'article n'est pas lu ou que les participantes démontrent très peu d'intérêt pour lui). Les trois premières réactions sont associées aux trois types de lectures de Stuart Hall (1973), soit les lectures « dominante », « oppositionnelle » ou « négociée ». Cette catégorisation de la réception en trois lectures a également été utilisée par Pierrette Bouchard et Natasha Bouchard (2003) dans leurs travaux sur l'hypersexualisation et les magazines. Nous y avons ajouté une quatrième, soit « l'ignorance », pour rendre compte du concept de la sélectivitét, concept selon lequel les récepteurs ont tendance à s'exposer aux contenus corroborant leur vision du monde et à ignorer (voire à modifier) les messages allant à l'encontre de leurs convictions ou de leurs comportements (Festinger, 1962 ; Hill, Watson, 2003 : 258 ; Balle, 1998 ; Barnouw, 1989 : 37 ; Baltes, Smelser, 2001 : 21 I2). II se pouvait aussi tout simplement que les participantes « ignorent » certains articles par manque d'intérêt, et que ce manque d'intérêt soit reflété, outre par la méthode crayon en main, dans les commentaires lors des entretiens. Comme la structure des entretiens a généralement suivi celle du contenu des revues (on a demandé d'entrée de jeu aux participantes de montrer comment elles lisaient les magazines et de commenter les articles), il a été aisé d'associer une réception (négociée, dominante, oppositionnelle ou ignorée) aux articles, chaque type de réception étant mutuellement exclusif. À l'inverse, les catégories des fonctions étaient nonexclusives, c'est-à-dire que plus d'une fonction pouvait être associée à chaque article dont il avait été question en entrevue.

\section{Résultats}

À l'instar de l'organisation de la problématique et des questions de recherche, les résultats se divisent en trois plans : ceux de l'intérêt, des fonctions et des diverses réceptions possibles.

\section{Plan I : l'intérêt suscité par les articles}

L'analyse du taux d'intérêt suscité par les articles a d'abord montré que les participantes lisent en effet les textes portant sur la sexualité, et qu'elles s'y intéressent. Ces textes sont lus dans une proportion de $54 \%$, dont $80 \%$ sont

\footnotetext{
${ }^{7}$ L'exposition sélective, souvent inconsciente, implique au départ qu'il y ait une perception sélective des messages, ce qui veut dire que les individus ont tendance à adopter une interprétation du message qui nuit le moins possible à leur représentation du monde. Cette supposition découle de la théorie de la dissonance cognitive, élaborée par L. Festinger (1962), et selon laquelle les gens sont portés à agir de la sorte pour diminuer leur dissonance interne et atteindre un certain confort, même passager (Hill,Watson, 2003 : 258 ; Balle, 1998 ; Barnouw, 1989 : 37 ; Baltes, Smelser, $2001: 2112)$.
} 
appréciés ${ }^{8}$. Les articles concernant les relations de couple ont été lus (59\%) et appréciés (82\%) dans une proportion un peu plus grande. Quant aux articles ou aux reportages traitant de mode et de beauté (qui, tout au long de cet article, constitueront notre comparatif), ils ont été lus à $49 \%$ et appréciés dans une proportion similaire aux autres catégories, soit à $81 \%$.

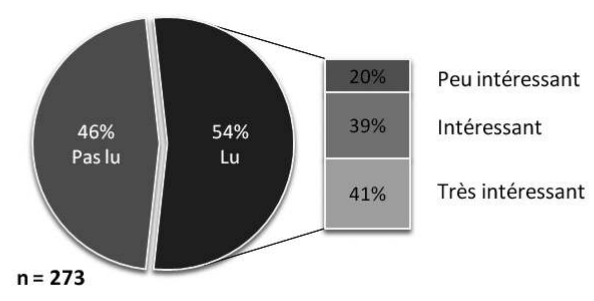

Figure I : Taux d'intérêt suscité par les articles traitant de sexualité.

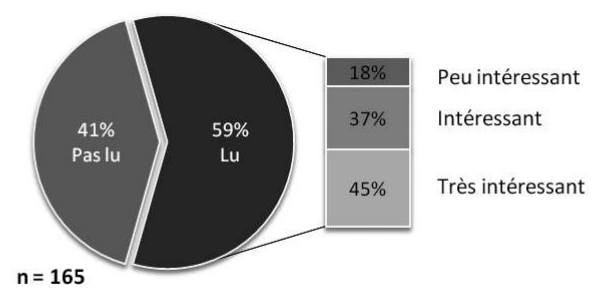

Figure 2 : Taux d'intérêt suscité par les articles traitant des relations de couple.

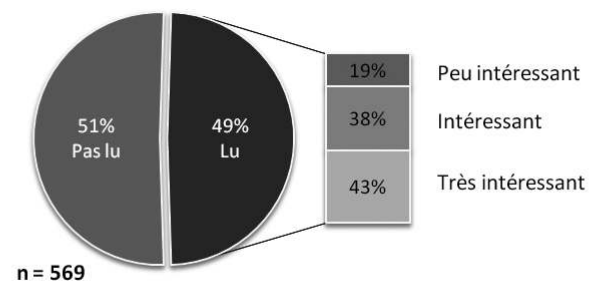

Figure 3 : Taux d'intérêt suscité par les articles traitant de mode et de beauté.

\footnotetext{
8 Pour cette appréciation, ont été considérés les articles jugés « intéressants 》 et « très intéressants »
} 


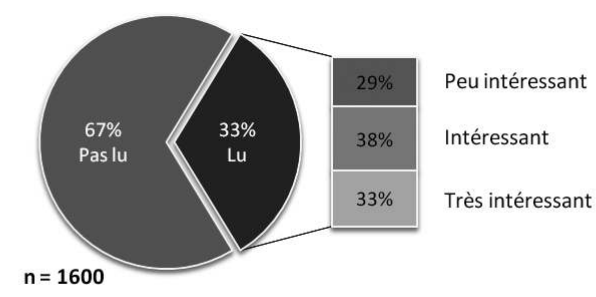

Figure 4 : Taux d'intérêt suscité par les articles traitant d'autres sujets.

Les articles traitant de thèmes moins fréquents (catégorie « autres ») sont lus dans une proportion significativement moindre, soit à $33 \%$. Toutefois, grâce à l'analyse du taux d'intérêt, il est possible de distinguer les thèmes de cette catégorie qui ont le plus intéressé les participantes, même si ces thèmes étaient peu présents dans les revues qui leur avaient été soumises. Les articles traitant de forme physique, de relations de couple, d'accomplissements faits par des modèles féminins et de la sexualité sont ceux qui, toutes catégories confondues, ont suscité le plus d'intérêt. Cependant, les articles traitant de forme physique ou présentant des exemples féminins sont très peu représentés dans la revue Adorable, soit quelques articles seulement par édition, contrairement aux articles qui traitent de mode, de beauté et de sexualité, qui couvrent une grande partie du contenu éditorial.

\begin{tabular}{|l|c|c|}
\hline \multicolumn{1}{|c|}{ Thèmes } & $\begin{array}{c}\text { Indice d'intérêt } \\
\text { (compris entre } \text { e et l) }\end{array}$ & Nombre d'articles \\
\hline Contenu éditorial de bas & 0.03 & 16 \\
\hline Actualité & 0.23 & 44 \\
\hline Décoration & 0.23 & 2 \\
\hline Technologie & 0.24 & 3 \\
\hline Stars & 0.28 & 27 \\
\hline Horoscope & 0.29 & 6 \\
\hline Mode & 0.29 & 13 \\
\hline Cuisine & 0.30 & 4 \\
\hline Consommation & 0.32 & 3 \\
\hline Psychologie & 0.32 & 2 \\
\hline Beauté & 0.39 & 27 \\
\hline Sexualité & 0.40 & 20 \\
\hline Exemple féminin & 0.42 & 4 \\
\hline Relations de couple & 0.45 & 12 \\
\hline Forme physique & 0.56 & 2 \\
\hline
\end{tabular}

Tableau I : Indice d'intérêt par catégorie.

\footnotetext{
${ }^{9}$ Le contenu éditorial de base comprend le sommaire, les éditoriaux, le courrier des lecteurs...
} 
Les articles traitant de sexualité se trouvent donc en $4^{\mathrm{e}}$ position en regard de l'intérêt ; ceux traitant de relations de couple, plus populaires, se trouvent en $2^{\mathrm{e}}$ position. Nous avons voulu savoir quels thèmes plus précis parmi ces catégories suscitaient le plus d'intérêt. Grâce à l'indice du taux d'intérêt, nous avons pu observer que les articles de type «Vox Pop » (soit articles décrivant des situations loufoques vécues par certaines lectrices), les articles traitant d'infidélité et ceux mettant de l'avant l'opinion des chroniqueurs masculins ont obtenu un taux d'intérêt élevé, suivis de près par les articles discutant du corps (en tant qu'attrait pour le sexe opposé) et ceux fournissant de l'information sexuelle (destinée à renseigner sur certaines pratiques). À l'opposé, les articles traitant des relations de couple (excluant l'infidélité) et des jouets sexuels ont obtenu un taux d'intérêt nettement moindre, de même que ceux présentant l'histoire de «l'homme sexy du mois » ou présentant une « lecture érotique $»^{10}$.

\section{Plan 2 : fonction des articles}

L'analyse du verbatim des entrevues a montré que les articles ont rempli trois fonctions différentes chez les participantes : elles ont lu les articles soit pour apprendre, soit pour le plaisir, et soit pour se rassurer quant à certains aspects de leur vie, que ces aspects soient actuels ou éventuels. Pour la très grande majorité des articles, la fonction qui est revenue le plus souvent est celle de l'apprentissage, c'est-à-dire que les textes ont eu pour elles un côté utilitaire : ils leur ont « donné des trucs », « fait remarquer » certaines choses, leur ont « ouvert les yeux », ou leur ont «montré comment faire ». Les textes sur la sexualité et le couple de même que ceux traitant de la mode et de la beauté ont largement été lus dans cette optique d'apprendre ou de se tenir au courant.Ainsi, sur toutes les fonctions recensées concernant les textes traitant de sexualité, $57 \%$ des occurrences sont-elles relatives à l'apprentissage ; similairement, $48 \%$ des fonctions recensées lors de la discussion des textes sur les relations de couple se rapportent à cette fonction. En comparaison, 67 \% des fonctions recensées lors de la discussion des textes « mode et beauté » se rapportent au fait d'apprendre quelque chose. La fonction « plaisir » prend également une place importante. Cette fonction a été recensée lorsque la participante mettait de l'avant le côté léger et ludique de sa lecture. Un peu à la manière des participantes de Janice Radway (1984), leur lecture était considérée « drôle », « le fun », « cocasse », « cute » ou « faisait rêver ». Là encore, les textes traitant de la sexualité et des relations de couple ont rempli grandement cette fonction (à $40 \%$ pour les deux catégories). En comparaison, $25 \%$ seulement des articles traitant de mode et de beauté ont été lus pour le plaisir. Ainsi, si tous les articles étudiés, tous thèmes confondus, leur servent majoritairement à apprendre, les textes à caractère sexuel sont-ils un peu plus lus pour le plaisir que ceux, par exemple, traitant de mode ou de

\footnotetext{
10 Par ailleurs, certaines catégories de textes n'ont suscité aucun intérêt chez les participantes qui les ont d'ailleurs souvent laissées sans marque de lecture. C'est le cas de presque toutes les publicités (que nous n'avons donc pas considérées), de même que des sections du magazine sans contenu « réel », comme le sommaire ou le carnet d'adresses.
} 
beauté. Cependant, les articles traitant des pratiques sexuelles sont plus lus pour apprendre (57\%) que ceux traitant des relations de couple (48\%). En troisième lieu, dans une moindre mesure, les textes leur ont servi à se « rassurer », c'est-àdire qu'ils contribuaient, selon les lectrices, à leur estime de soi et à l'acceptation de leur corps. Cela signifiait souvent, par exemple, que la participante avait réalisé qu'elle n'était pas « la seule dans cette situation ». Cette fonction, quoique bien présente, a été généralement peu fréquente (3\% pour la sexualité, 12 \% pour les relations de couple et $8 \%$ pour mode-beauté). Cependant, l'analyse des thèmes précis auxquels était associée cette fonction a démontré que ce ne sont pas les relations de couple en elles-mêmes qui inquiètent les participantes, mais bien certains aspects particuliers de la vie de couple. Ainsi $14 \%$ des fonctions recensées lors des articles traitant de l'infidélité concernent-elles le fait de se rassurer, alors que la proportion tombe à zéro dans tout autre thème touchant le couple, sauf un. En effet, les occurrences de fonctions illustrant le besoin de rassurance montent en flèche lorsqu'il est question du corps (33\%). II semble donc que si les jeunes participantes semblaient préoccupées par l'apparence de leur corps (dans le but éventuel de séduire les garçons), elles n'avaient pas encore besoin d'être rassurées par rapport au couple (sauf en ce qui concerne certaines craintes liées à une éventuelle infidélité).

\section{Plan 3 : réception des articles}

Comme nous l'avons mentionné plus haut, nous avons distingué quatre types de réception : les réceptions dominante, négociée et oppositionnelle, puis l'ignorance. On a considéré que la participante faisait une réception dominante d'un article lorsqu'elle était d'accord avec le texte étudié et qu'elle n'y soulevait aucune opposition. Ceci s'est traduit par le fait d'accorder beaucoup de crédibilité au texte, parfois plus qu'à elle-même. Une réception négociée signifie que la participante est partagée entre deux opinions. Elle peut trouver l'article intéressant, mais elle exprime des craintes ou ne l'endosse pas complètement. La réception oppositionnelle, quant à elle, illustre les cas où le discours tenu par la participante s'oppose directement au contenu de l'article. L'ignorance, enfin, réfère aux cas où la participante illustre un faible niveau d'intérêt pour l'article qui lui semble ennuyeux (les commentaires recueillis pour ce type de réception ont également servi à peaufiner et à confirmer l'analyse du taux d'intérêt, expliquée précédemment). II est important de préciser que le fait de démontrer une réception dominante d'un texte n'équivaut pas nécessairement à un manque d'esprit critique, de la même façon qu'un article figurant dans un magazine pour adolescentes n'est pas systématiquement «mauvais »"'. La réception dominante n'est donc pas toujours naïve, comme le démontrent ces deux participantes discutant d'une chronique masculine qu'elles ont trouvée bien écrite :

\footnotetext{
"Pour reprendre les termes de certaines analyses de contenu antérieures, tous les articles ne contiennent pas systématiquement des prescriptions idéologiques « néfastes » pour les filles. Certains peuvent par exemple mettre de l'avant des valeurs de respect de soi-même et de ses partenaires.
} 
«- Je suis d'accord avec le gars dans ce cas-ci (Catherine ${ }^{12}$ )

- Moi aussi. Je trouvais qu'il n'était pas le macho qui veut la petite raide maigre un peu nounoune ${ }^{13}$,

là (Jessica) ».

Le but de la distinction des réceptions n'est donc pas d'émettre un jugement de valeur sur la capacité des participantes à émettre des commentaires critiques, mais bien de comprendre les mécanismes de réception qui sous-tendent la lecture du magazine pour en voir les implications. Lors de l'analyse des résultats du plan 3, nous avons remarqué que les articles étaient généralement reçus de façon dominante, soit à 42 \% (c'est-à-dire 268 articles reçus de façon dominante sur un total de 635 articles discutés lors des entrevues ${ }^{14}$ ). Cependant, en comparaison avec les articles traitant de la mode et de la beauté, moins d'articles portant sur la sexualité et les relations de couple ont été reçus de façon dominante (37\% pour la sexualité et les relations de couple contre $48 \%$ pour la mode-beauté). $15 \%$ des articles traitant de sexualité et $20 \%$ des articles sur les relations de couple ont été reçus de façon négociée, alors que respectivement 15 et $12 \%$ de ceux-ci ont été reçus de façon oppositionnelle. On obtient des résultats similaires dans la catégorie « Mode-beauté », soit 16 \% d'articles reçus de façon négociée et $13 \%$ de façon oppositionnelle. Dans chacune des catégories traitant de couple et de sexualité, environ le tiers des articles discutés lors des entrevues ont été ignorés par les participantes, c'est-à-dire qu'elles ont dit les trouver ennuyeux ou avoué spontanément ne pas les avoir lus ${ }^{15}$. Un peu moins d'articles traitant de mode et de beauté ont été ignorés, soit $23 \%$.

\section{Dominante}

Ignorée

Négociée
Oppositionnelle

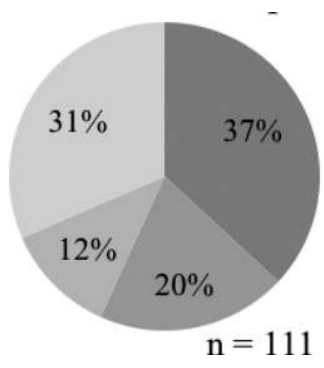

Figure 5 : Réception des textes de la catégorie « Relations de couple ».

12 Les noms ont été changés.

13 Ici, nounoune signifie naïve.

14 Le pourcentage concerne la proportion totale des articles discutant de mode, de beauté, de sexualité et de relations de couple ayant été reçue de façon dominante. Les autres thèmes (forme physique, exemples féminins, etc.) n'ont pas été considérés à cette étape de l'analyse, qui avait pour but de décrire la réception des textes à caractère sexuel. La catégorie « Mode-Beauté » sert cependant toujours ici de comparatif.

15 |l est à noter que tous les articles n'étaient pas systématiquement discutés lors des entretiens ; c'était le cas notamment des articles encerclés en bleu ou en vert ou des articles n'entrant pas dans les catégories principales de la sexualité, des relations de couple ou de la mode et de la beauté. 


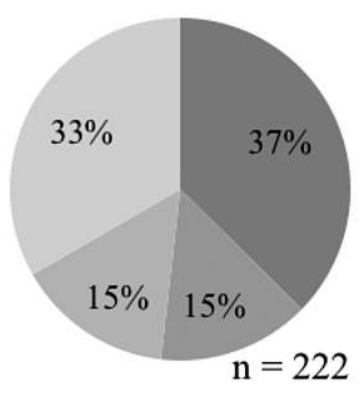

Figure 6 : Réception des textes de la catégorie « Sexualité ».

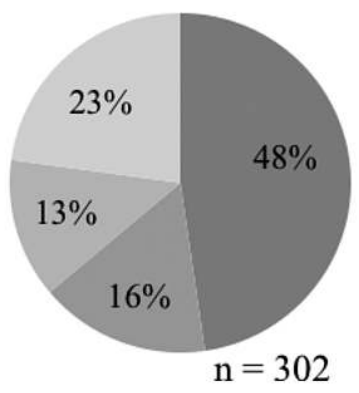

Figure 7 : Réception des textes de la catégorie « Mode-Beauté ».

Lorsqu'on analyse plus en détail les thèmes reçus de façon dominante, on remarque que les articles traitant du corps (50\%), présentant de l'information sexuelle à visée éducative (48\%), discutant de l'infidélité (46\%) ou utilisant la formule «Vox Pop » (85\%) ont obtenu le plus haut taux de réceptions dominantes. À l'inverse, les articles portant sur l'érotisme ou proposant une lecture érotique sont ceux qui ont soulevé le plus d'opposition, toutes proportions gardées (réception oppositionnelle à $28 \%$ ). Les articles présentant ou discutant des jouets sexuels, bien qu'ils aient majoritairement été reçus de façon dominante, présentent aussi une forte proportion de réceptions oppositionnelles (dominance : 36 \% ; négociation : $17 \%$; opposition : $25 \%$ ). Ce sont toutefois les chroniques masculines qui ont le plus fait l'objet de réceptions mitigées : elles ont suscité autant de réceptions dominantes que de réceptions négociées ou oppositionnelles (30 \% chacune). Ce sont d'ailleurs souvent les chroniques masculines ou les textes écrits par des hommes qui ont reçu les critiques les plus acerbes. L'article « Le corps parfait, le revers de la médaille... », écrit par un homme, a notamment soulevé beaucoup d'objection, comme le démontrent ces deux amies, qui discutent entre elles : 
«- C'est encore écrit par Stéphane Martel, et moi je le porte pas vraiment dans mon cœur. [...] (Émilie)

- Moi, j'avoue qu'on dirait que cet auteur-là, il pense qu'il a raison dans tout ce qu'il écrit. [...] (Audrey)

- Je ne sais pas s'il écrit pour donner une leçon [aux femmes], parce que c'est une revue de femmes, mais... (Émilie)

- Il est vraiment provocateur. [...] (Audrey)

- Je suis certaine qu'on lirait plein d'articles différents de lui, et qu'il y a[urait] plein de fois [où] il se contredi[rait]... [...] Ça dépend de comment il se sent, là, dans le mois... (Émilie)

- Ah, c'est ça, «Aujourd'hui, je m'attaque aux femmes ! »[rires] (Audrey) ».

Cet extrait démontre bien la façon dont les participantes se sont opposées à plusieurs textes portant sur la sexualité.

\section{Conclusion}

Comme cela avait été le cas dans l'étude de Dawn H. Currie (1999), les participantes de notre étude ont majoritairement adhéré au contenu du magazine Adorable, puisque la réception qui en a été faite a généralement été dominante (48\% pour « Mode-beauté » et $37 \%$ pour « Sexualité » et « Relations de couple »). Bien que les participantes aient estimé que les articles traitant du corps et de l'infidélité contribuaient à les rassurer, elles ont aussi montré, par leurs commentaires, que ces deux thèmes constituaient ceux qui suscitaient chez elles le plus d'inquiétude. Dès lors, il est difficile de postuler sur le véritable effet des articles qui traitent de ces deux thèmes : des études antérieures, par exemple, ont montré que l'exposition à des mannequins minces et attrayants dans les médias avait pour effet d'augmenter l'insatisfaction des jeunes femmes par rapport à leur corps et de diminuer leur confiance (Ward, Harrison, 2005 : 9 ; Wykes, Gunter, 2005). D'autres encore ont montré que les médias qui encouragent l'idée d'une sexualité récréative et les rôles stéréotypés traditionnels participent à augmenter chez les jeunes filles une vision négative des relations de couples (Ward, Harrison, ibid. : 12). Selon ces derniers auteurs, les médias semblent agir, en ce qui concerne la sexualité, comme de puissants agents socialisants, qui peuvent affecter les perceptions que les réceptrices peuvent avoir d'elles, de leur corps et de leur entourage (ibid. : 14), et ce, particulièrement lorsque celles-ci sont jeunes, en quête d'idéaux et en processus d'apprentissage (Wykes, Gunter, ibid. : 148). Or, dans notre étude, les participantes utilisent les textes qui traitent de la perfection du corps pour se rassurer (elles étaient soulagées qu'un chroniqueur masculin dise aimer les filles « enrobées »), alors il est difficile de savoir si Adorable (qui regorge de photos de femmes très peu vêtues et aux courbes parfaites) les aide plus qu'il ne les nuit à ce propos.

Chose certaine, le contenu les amène à discuter de ces images, et les chroniques, lorsqu'elles sont bien écrites ou, au contraire, désolantes, les amène à confronter leurs valeurs avec celles des auteurs. Au mieux, elles y gagnent en perspective ou 
réaffirment leurs positions, et dans le pire des cas, elles apprennent à savoir à quoi s'attendre des hommes les plus machos. D'ailleurs, les résultats montrent que les participantes ont majoritairement lu les articles (qu'ils soient à caractère sexuel ou non) dans la perspective d'apprendre, ce qui corrobore aussi les résultats d'autres études qui montrent que les médias peuvent jouer un rôle important dans l'apprentissage de la sexualité chez les sujets adolescents (Steele, 1999 ; Brown, 2002). Cependant, plusieurs des articles à visée « éducative » contenus dans les magazines pour adolescentes tendent généralement à dépeindre une vision sexiste, hétéronormative et stéréotypée de la sexualité (Caron, 2003b). Par ailleurs, de l'aveu d'une journaliste « sexo », certains articles discutant de nouvelles «tendances » sexuelles n'ont pas nécessairement de fondements véridiques. Par exemple, Anne Steiger (2006) a ouvertement avoué avoir inventé de toutes pièces certaines de ces «tendances ». Or, si l'on peut déplorer le fait qu'une grande partie du magazine met l'accent sur la sexualité récréative et qu'elle présente celle-ci de façon normative et peu « égalitaire $»^{16}$, on ne peut négliger la part des résultats, bien présente, démontrant des réceptions oppositionnelles et négociées des contenus les plus « extrêmes ». Les articles proposant un extrait d'une nouvelle érotique ont été jugés exagérés et ridicules ${ }^{17}$; les articles faisant la promotion de jouets sexuels, bien qu'ils aient été reçus négativement par moins de participantes, ont toutefois reçu des critiques encore plus acerbes par celles qui les rejetaient. Bref, malgré des allers-retours complexes dans les réceptions des articles, il semble d'une manière générale que les participantes adhèrent aux discours dominants du contenu, mais qu'elles rejettent tout ce qui leur semble dépasser une certaine limite acceptable. Cette « limite » est construite en premier lieu par leurs valeurs (elles se prononcent contre l'infidélité et contre la sexualité débridée et déconnectée des sentiments), et ensuite par la reprise d'un discours féministe ambiant (les conseils des chroniqueurs masculins sont rejetés, étant perçus comme motivés par un sentiment de supériorité qui ne passe pas). Ainsi, malgré la forte propension des participantes à adhérer à l'idéologie dominante de la revue, ont-elles montré qu'elles étaient capables de faire preuve d'esprit critique à certaines occasions : elles ne « gobent » pas tout ce qu'elles lisent sans jauger la qualité des textes, les opposer à leurs valeurs ou en questionner les sources et les fondements. Ce constat corrobore ceux d'Elizabeth Frazer ( 1987), de Dawn H. Currie (1999), Lisa L. Duke et Peggy J. Kreshel (1998) puis, dans une plus petite mesure, ceux de Pierrette Bouchard et Natasha Bouchard (2005) qui ont montré que, malgré leur adhésion au discours dominant des textes, les lectrices n'en sont pas pour autant « victimes » mais, qu'au contraire, elles peuvent en négocier les significations. En effet, toutes nos participantes ont été capables de faire preuve d'esprit critique à différentes occasions par des

\footnotetext{
16 «Égalitaire » est le terme employé par le Conseil du statut de la femme de la province de Québec (2008) qui fait référence à une sexualité qui mise sur le respect de soi et des autres, le tout dans une perspective d'égalité des genres.

${ }_{17}$ Ce qui n'empêche pas le fait que les extraits ont été trouvés très drôles, ce qui démontre la complexité de la réception des contenus et la négociation des significations initiales, à l'image des lectrices de J. Radway (1984).
} 
réceptions oppositionnelles, et plusieurs ont insisté sur le fait qu'elles comptaient conserver leurs opinions premières malgré l'incitation de certains textes à les changer.

Comme le souligne Éric Maigret (1995: 98) dans son étude de réception des bandes dessinées chez les garçons, lorsque l'apprentissage de la sexualité (ou, dans son cas, des identités sexuelles) se fait par les médias, c'est par le biais de relations complexes; il ne repose pas sur « de simples instances de reproduction des caractéristiques sexuelles, de perpétuation d'un ordre traditionnel », mais sur un « travail de construction » (ibid. : 99) important souvent accompagné de conversion des valeurs originelles des contenus. Dans notre étude, les propos des participantes sont constitués de plusieurs discours opposés, voire contradictoires ; elles construisent leurs propres interprétations, et celles-ci sont amenées à évoluer constamment au fil des lectures. Enfin, il ne faut pas oublier que la réception chez les enfants ou les adolescents peut être influencée par plusieurs critères extérieurs, tels l'encadrement familial, la scolarité des parents, la personnalité, l'expérience du sujet, etc. (voir notamment Singer, Singer, 200 I : 297-298 ; Kundanis, 2003 ; Strasburger,Wilson : 2002) qui peuvent jouer un rôle important dans l'élaboration de ces interprétations. Par exemple, une participante a utilisé l'expérience de sa famille pour justifier la réception dominante d'un texte traitant d'infidélité : un membre de sa famille ayant été infidèle, elle disait réaliser que l'infidélité se produisait dans des contextes souvent difficiles, et qu'ainsi il valait mieux ne pas juger d'emblée les gens infidèles. Ce dernier exemple révèle toute la complexité du processus de réception de même que l'importance de reconnaître aux adolescents la capacité d'interprétation et de négociation des contenus. Le discours sur la sexualité de la revue Adorable constitue un discours parmi d'autres; or, selon Michel Foucault (1972), ce sont les discours les plus puissants qui réussissent à s'établir comme des « vérités ». Bien que nos participantes aient reçu les contenus d'Adorable de façon généralement dominante, le discours de la revue ne semblait pas le plus fort. Leurs valeurs (probablement issues des discours familiaux) semblaient prendre une place plus importante. Ce qui ne veut pas dire qu'on ne puisse toutefois souhaiter que les contenus sexuels des médias disponibles aux adolescents et aux adolescentes présentent une qualité qui encourage un peu plus le respect de soi et des autres, de même que les « rapports égalitaires » (Conseil du statut de la femme, 2008). Une précaution qui gagnerait à être couplée à l'assurance d'une bonne éducation des jeunes filles et des garçons quant au contenu des médias qui leur sont destinés. 


\section{Références}

Balle F., dir., 1998., Dictionnaire des médias, Paris, Larousse.

Baltes P.-B., Smelser N.-J., eds, 200I, International Encyclopedia of the Social \& Behavioral Sciences, Amsterdam/New York, Elsevier.

Barnouw E., ed., 1989, International Encyclopedia of Communications, New York, Oxford University Press.

Bouchard, P., Bouchard N., 2003, «"Miroir, miroir..." : La précocité provoquée de l'adolescence et ses effets sur la vulnérabilité des filles », Les Cahiers de recherche du GREMF, 87, pp. 5-24.

- 2005, « L'imprégnation idéologique et la résistance : étude des réactions d'un groupe de préadolescentes à deux magazines pour jeunes filles », Recherches féministes, | 8, I, pp. 5-24.

Brown J.-D., 2002, « Mass Media Influences on Family », The Journal of Sex Research, 39, I, pp. $42-45$.

Caron C., 2003a, « Introduction aux travaux de langue anglaise sur la presse féminine (1978-200 I) », Les Cahiers de recherche du GREMF, 19, pp. 49-6I.

- 2003b, La presse féminine pour adolescentes : une analyse de contenu, Mémoire de maîtrise en communication publique, Université Laval.

- 2007, «Adolescentes et discours médiatiques québécois : des corps omniprésents, mais des voix inaudibles » (communication orale), Chaire pour le développement de la recherche sur la culture d'expression française en Amérique du Nord, Québec, Université Laval, 5 oct.

- 2009, « Les adolescentes dans le discours médiatique québécois : une présence paradoxale », pp. 205-221, in : Brun J., dir., Interrelations femmes-médias dans l'Amérique française, Québec, Presses de l'université Laval.

Conseil du statut de la femme, 2008, Le sexe dans les médias : obstacle aux rapports égalitaires, Québec. Accès : www.csf.gouv.qc.ca/fr/publications.

Currie D.-H., 1999, Girl Talk : Adolescent Magazines and Their Readers, Toronto, Presses de l'université de Toronto.

Cyr M.-F., 1998, Parades et modèles de relations homme-femme dans les magazines féminins québécois de 1993, Thèse de doctorat en communication, Université du Québec à Montréal.

Davis, H., 2004, Understanding Stuart Hall, London, Thousand Oaks / New Delhi, Sage Publications.

Debras S., 2003, Lectrices au quotidien : femmes et presse quotidienne, la dissension, Paris, Éd. L'Harmattan.

Duke L.-L., Kreshel P.-J., 1998, « Negociating Feminity : Girls in Early Adolescence Read Teen Magazines », Journal of Communication Inquiry, 22, I, pp. 48-71.

Festinger L., 1962, A Theory of Cognitive Dissonance, Stanford, Stanford University Press.

Foucault M., 1972, The Archeology of Knowledge, London, Tavistock Publications. 
Francoeur R.-T., Noonan, R.-J., eds, 2004, The Continuum Complete International Encyclopedia of Sexuality, New York, Continuum.

Frazer E., 1987, «Teenage Girls Reading Jackie », Media, Culture and Society, 9, 4, pp. 407425.

Hall S., 1980, « Coding, Decoding », pp. 907-916, in : Ryan M., ed., Cultural Studies : An Anthology, Malden, Oxford, Carlton, Blackwell Publishing, 2008.

2007, « Cultural Studies and its Theoretical Legacies », pp. 33-44, in : During S., éd., The Cultural Studies Reader, $3^{e}$ éd., London/New York, Routledge.

Hermes J., 1995, Reading Women's Magazines: An analysis of Everyday Media Use, Cambridge, Polity Press.

Hill A., James W., 2003, Dictionary of Media and Communication Studies, London, Arnold / New York, Oxford University Press.

Kissling E.-A., 1996, « Bleeding Out Loud: Communication about Menstruation », Feminism \& Psychology, 6, 4, pp. 48I-504.

Kundanis R.-M., 2003, Children, Teens, Families, and Mass Media : The Millennial Generation, Mahwah (New Jersey), Lawrence Erlbaum Associates.

Lang M.-E., 2009, La réception des textes de magazines à caractère sexuel chez adolescentes au Québec et au Nouveau-Brunswick : adhésion, ignorance ou contestation?, Mémoire de maîtrise en communication publique, Université Laval.

Laperrière A., 1997, « Les critères de scientificité des méthodes qualitatives 》, pp. 365-389, in : Deslauriers P., Groulx L., Laperrière A., Mayer R., Pires A., Poupart J., dirs, La recherche qualitative : enjeux épistémologiques et méthodologiques, Montréal, G. Morin.

Lazarsfeld P.-F., Berelson B., Gaudet H., 1968, The People's Choice : How the Voter Makes Up His Mind in a Presidential Campaign, New York, Columbia University Press, 3 e éd.

Lebel E., 2004, « Lectrices au quotidien », Cahiers du journalisme, I3, pp. 388-39|.

Maigret É., 2005, «"Strange grandit avec moi". Sentimentalité et masculinité chez les lecteurs de bandes dessinées de super-héros », Réseaux, vol. | 3, 70, pp. 79- 03.

McRobbie A., 1982, « Jackie : An Ideology of Adolescent Feminity », pp. 263-283, in : Bennet T., Martin G., Waites B., dirs, Popular Culture : Past and Present, London, Croom Helm.

Radway J., 1984, Reading the Romance: Women, Patriarchy, and Popular Literature, Chapel Hill/London, University of North Carolina Press.

Rakow L.-F., Wackwitz L.-A., 2005, Feminist Communication Theory Selections in Context, Thousand Oaks, Sage Publications.

Singer D.-G., Singer J.-L., 200 I, Handbook of Children and the Media, Thousand Oaks Sage Publications.

Steele J.-R., 1999, «Teenage Sexuality and Media Practice: Factoring in the Influences of Family, Friends, and School », The Journal of Sex Research, 36, 4, pp. 33I-34I.

Steiger A., 2006, La vie sexuelle des magazines : comment la presse manipule notre libido et celle des ados, Paris, Michalon.

Stevenson N., 2002, Understanding Media Cultures : Social Theory and Mass Communication, London, Sage Publications, $2^{e}$ éd. 
Strasburger V., Wilson B.--., 2002, Children, Adolescents and the Media, Cambridge, Polity Press in association with Blackwell Publishers.

Trudeau V., 2007, « Nouvelle union! », Adorable, juin, 124, p. 4.

Van Zoonen L., 1994, Feminist Media Studies, London, Sage Publications.

Voirol O., 2005a, «Présentation du dossier "Visibilité/lnvisibilité" », Réseaux, |29-|30, pp. 9-35.

- 2005b, « Les luttes pour la visibilité : esquisse d'une problématique », Réseaux, | 29- | 30, pp. 89-12I.

Ward L.-M., Harrison K., 2005, «The Impact of Media Use on Girls' Beliefs about Gender Roles, their Bodies, and Sexual Relationships: A Research Synthesis », pp. 3-23, in : Cole E., Daniel J. H., eds, Featuring Females : Feminist Analysis of Media, Washington, DC, American Psychological Association.

Wykes M., Gunter B., 2005, The Media \& Body Image, London, Thousand Oaks/New Delhi, Sage Publications. 